\title{
Skull Base 3D Modeling of Rigid Buttress for Gasket-Seal Closure Using Operative Endoscopic Imaging: Cadaveric Feasibility
}

\author{
James Shin ${ }^{1}$ Jonathan Forbes ${ }^{2}$ Kurt Lehner $^{3}$ Hilarie Tomasiewicz ${ }^{2}$ Theodore H. Schwartz ${ }^{2}$ \\ C. Douglas Phillips ${ }^{1}$
}

${ }^{1}$ Department of Radiology, Weill Cornell Medicine, Cornell University, New York, New York, United States

2 Department of Neurosurgery, NewYork-Presbyterian/Weill Cornell

Address for correspondence James Shin, MD, MSc, Department of Radiology, Weill Cornell Medicine, 525 E 68th Street, New York, NY Medicine, New York, New York, United States

${ }^{3}$ Donald and Barbara Zucker School of Medicine at Hofstra/Northwell, Hofstra University, Hempstead, New York, United States

J Neurol Surg B 2019;80:67-71.

\begin{abstract}
Keywords

- photogrammetry

- gasket-seal

- buttress

- sellar reconstruction

- transsphenoidal

Surgical defect closure following endonasal transsphenoidal tumor resection is a critical component of procedural success. Three-dimensional (3D) modeling of relevant skull base anatomy during resection can potentially facilitate design of a custom rigid buttress for gasket-seal closure; however, access to conventional cross-sectional imaging intraoperatively is limited and cumbersome. Endoscopic imaging, by contrast, is always available. This work demonstrates the feasibility of 3D modeling of the visible skull base through structure-from-motion photogrammetric postprocessing techniques, providing a suitable template to design a gasket-seal buttress. Additionally, endoscopic 3D reconstruction of skull base surface anatomy may represent a more robust depiction of the surgical defect than is available by conventional 3D modeling with computed tomography, which suboptimally recapitulates very thin bones and mucosal surfaces typical of this regional anatomy.
\end{abstract}

\section{Background}

Skull base reconstruction following endonasal transsphenoidal tumor resection is a focus of active technical development that has seen steady evolution of multiple approaches to surgical defect closure over the past decade. ${ }^{1-11}$ A primary consideration during sellar floor reconstruction is the adequacy of seal around the defect, which may be implicated in rates of postoperative cerebrospinal fluid leak. ${ }^{1,9,12,13}$ At our institution, the gasket-seal method is the preferred closure technique following expanded endonasal approach for transsphenoidal tumor resection ${ }^{1}$ however, this technique requires intraoperative preparation of a semirigid buttress (MedPor or autograft), manually fashioned by subjective visual assessment of the surgical defect on endoscopic image, with iterative

received

February 10, 2018

accepted

June 2, 2018

published online

July 20, 2018 revisioning. ${ }^{1,10,11}$ As defect closure is performed immediately following resection, fabrication of an implantable rigid gasketseal buttress derived from operative imaging and deliverable within a narrow time window requires substantial workflow optimization paramount to eventual feasibility in practice.

This work details our experience establishing a passive monocular photogrammetric image post-processing pipeline to provide dimensionally accurate three-dimensional (3D) reconstructions of the visible skull base using conventional operative endoscopic imaging, to facilitate design and eventual fabrication of a rigid gasket-seal buttress. Although photogrammetry in medicine already spans numerous and varied applications across specialties, with the exception of ophthalmologic applications externally visible anatomy is ๑) 2019 Georg Thieme Verlag KG
Stuttgart · New York
DOI https://doi.org/ 10.1055/s-0038-1667023. ISSN 2193-6331. 
typically the anatomic focus. ${ }^{14-16}$ More recently, photogrammetric processing has been applied to endoscopic imaging for targeted applications. ${ }^{17-21}$ To our knowledge, this is the first application of these techniques to endonasal skull base imaging using unmodified, conventional endoscopic hardware.

\section{Methods}

A sellar floor defect was prepared on a cadaveric subject in standard fashion. A calibration object comprised of a noncoded $2 \mathrm{~mm}$ checkered squares pattern was placed adjacent to the defect endonasally. High-definition video $(1920 \times 1088$ pixels $)$ was recorded using a standard endonasal telescope (Karl Storz GmbH \& Co. KG). Using a retail Macbook Pro (7th generation 2.9GHz i7, 16GB RAM, 4GB AMD Radeon Pro 560), video clips were separated into individual frames at progressive batch-reduction rates. Image sets were processed into a 3D mesh model using the structure-from-motion (SFM) photogrammetry software (Agisoft PhotoScan v1.3), using shared processing parameters (high accuracy alignment, medium quality dense cloud generation, high quality mesh generation, aggressive depth filtering). Calibration object marker points were automatically detected, and the mesh model was scaled to match known dimensions prior to export in polygon file format (.ply). A planar buttress was modeled to fit the defect in Autodesk Meshmixer, using contour and vertex color information to determine the defect margin visually. The planar buttress was arbitrarily thickened to $0.6 \mathrm{~mm}$. Postprocedure computed tomographic (CT) images of the cadaveric subject were acquired, and a standard 3D bone model was generated for comparison. Visible skull base surface landmarks were used to manually coregister the endoscopic mesh model to the CT bone model for comparison.

\section{Results}

A 35-second endoscopic video clip was separated into 1,063 frames. All image batches yielded visually interpretable and dimensionally interrogable mesh models. Progressive batch reduction rates, total image-to-mesh processing times, and mean calibration errors are provided in (-Table 1) and (-Fig. 1). Results of sparse point cloud generation, dense point cloud generation, per-image camera positioning, and calibration marker point detection are depicted in (-Fig. 2 ). Increasing batch size was associated with longer processing times and more frequent image exclusions on the basis of automated alignment failure. Mean calibration errors reached a maximum error of $0.45 \mathrm{~mm}$ resulting from the largest batch (532 images, 50\% total), and a minimum error of $0.04 \mathrm{~mm}$ resulting from a batch of 107 images (10\% total). A planar buttress was created using the mesh model template derived from the latter image set, measuring $9.37 \times 6.65 \times 0.6 \mathrm{~mm}$. Coregistered endoscopic and CT skull base models with buttress in place are depicted in (-Fig. $\mathbf{3}$ ). Calculated depth maps and average reprojection error vectors for each image set are provided in (-Fig. 4).
Table 1 Image-to-mesh postprocessing times and calibration error for each image set

\begin{tabular}{|l|l|l|l|l|}
\hline $\begin{array}{l}\text { Batch } \\
\text { reduction }\end{array}$ & Images & $\begin{array}{l}\text { Aligned } \\
\text { images }\end{array}$ & $\begin{array}{l}\text { Processing } \\
\text { time }(\text { min) }\end{array}$ & $\begin{array}{l}\text { Calibration } \\
\text { error }(\mathbf{m m})\end{array}$ \\
\hline $1: 120$ & 10 & 10 & 0.50 & 0.20 \\
\hline $1: 60$ & 19 & 19 & 1.33 & 0.14 \\
\hline $1: 30$ & 36 & 35 & 3.67 & 0.11 \\
\hline $1: 20$ & 54 & 53 & 10.00 & 0.12 \\
\hline $1: 10$ & 107 & 105 & 25.67 & 0.04 \\
\hline $1: 50$ & 213 & 90 & 53.00 & 0.33 \\
\hline $1: 40$ & 267 & 137 & 56.50 & 0.31 \\
\hline $1: 30$ & 355 & 133 & 79.00 & 0.41 \\
\hline $1: 20$ & 532 & 300 & 185.50 & 0.45 \\
\hline
\end{tabular}

Note: Batch reductions are relative to a total of 1,063 images, extracted from a 35-second endoscopic video clip.

\section{Discussion}

These results demonstrate that relatively sparse optical image data can yield a dimensionally adequate 3D rendering of the skull base, serviceable as a template for gasket-seal buttress modeling. While ultra-wide/fish-eye endoscopic cameras and a small surgical field are inherently suboptimal for this method of SFM photogrammetry, this can be mitigated to an extent by oversampling (i.e., larger batch size) and
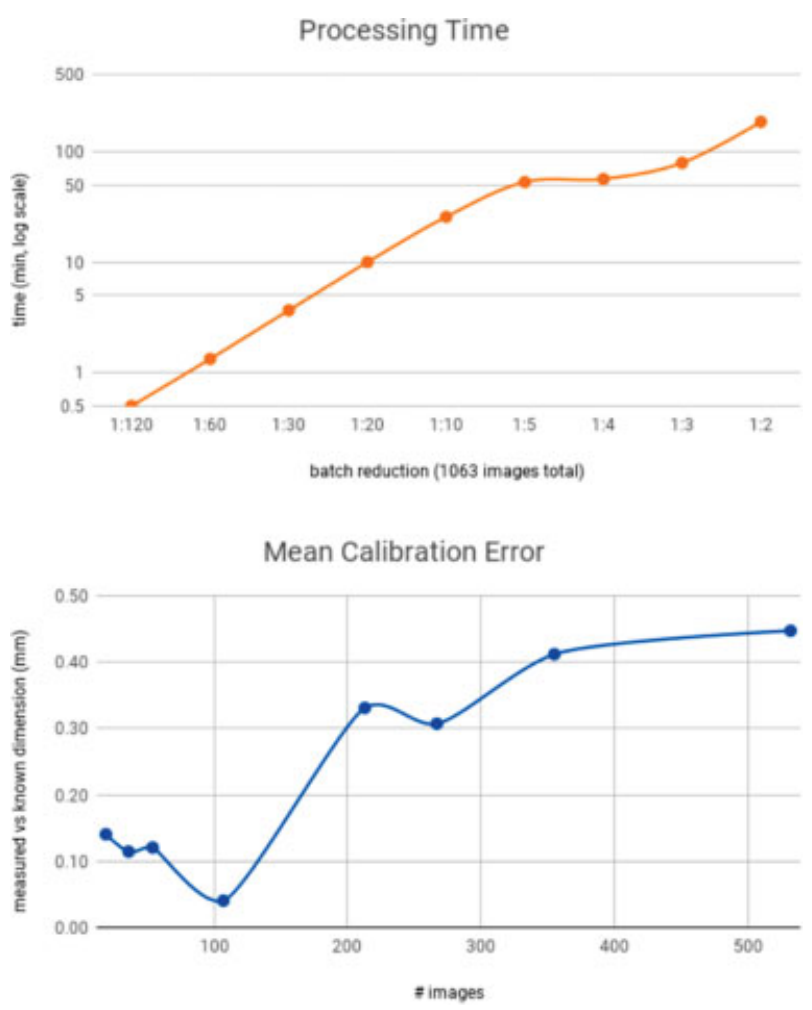

Fig. 1 Image-to-mesh postprocessing times and mean calibration errors for each image batch. Note the logarithmic scale corresponding to total processing time. 


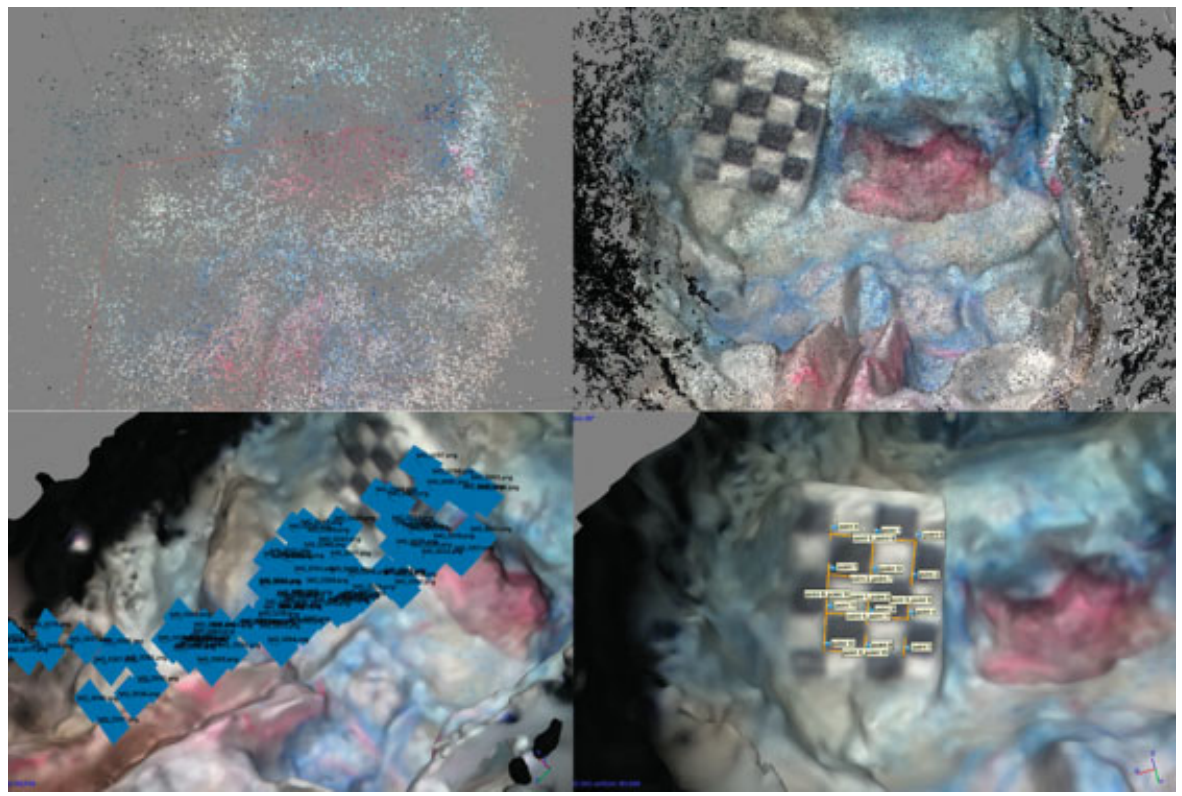

Fig. 2 Stepwise results of photogrammetric processing of endoscopic image data including (from top left): sparse point cloud generation, dense point cloud generation, per-image camera positioning, and calibration marker point detection.

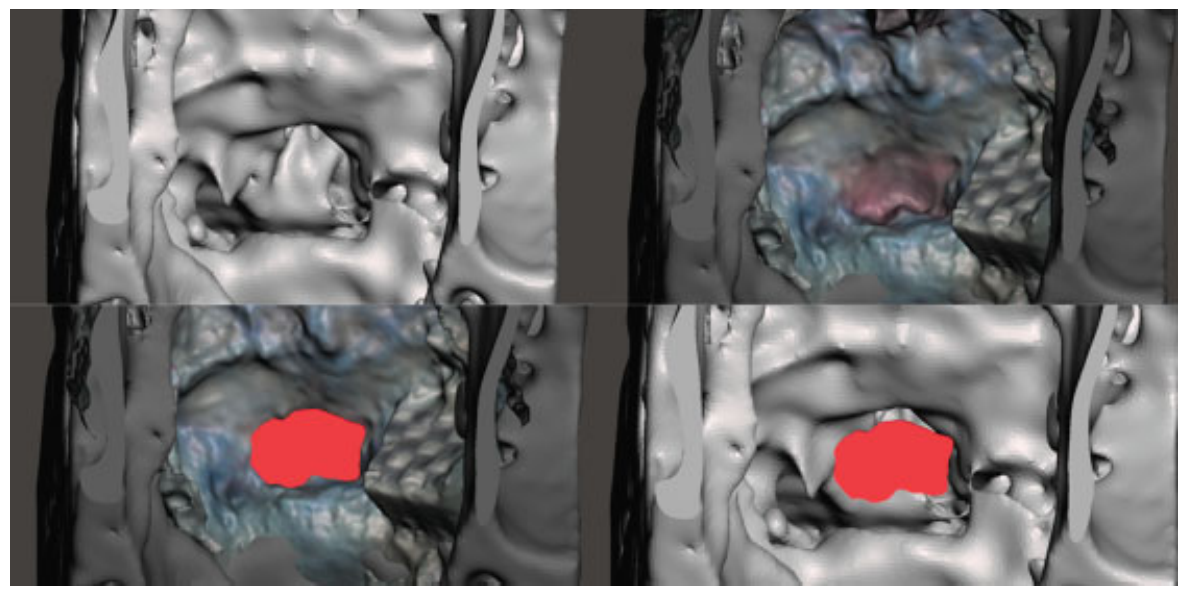

Fig. 3 En-face views of sellar floor defect (from top left) as seen on: computed tomography (CT) bone model, CT bone model with endoscopic mesh, CT bone model with endoscopic mesh and planar buttress, and CT bone model with planar buttress. Note the artifactual uncovering of the internal carotid arteries bilaterally and suboptimal reproduction of defect margins. By contrast, note the preservation of both surface contour and color detail indicating defect margins on the endoscopic mesh.

lens precalibration testing. This is a computationally expensive workflow to undertake, however, and very large batch sizes (i.e., $>100$ images) were encumbered by long processing times and larger calibration errors compared with smaller batch sizes. Examining reprojection error vectors across each image set ( $\boldsymbol{- \text { Fig. }}$ 4) reveals concentration of errors in the periphery of the frame-a result of geometric lens distortion. These errors, as well as the rate of image nonalignment ( - Table 1 ), increased substantially beyond a batch size of approximately 100 images in this configuration.

An increase in processing time with a larger batch size was expected and is amenable to hardware speed optimizations such as multigraphics processing unit. These findings suggest that this postprocessing workflow can be optimized to meet a narrow intraoperative window between defect creation and closure. An apparent reduction in dimensional fidelity at the largest batch sizes was unanticipated. This may be attributable to myriad and likely multifactorial causes-for example, more frequent inclusion of motion-degraded or off-focus images, more frequent intraframe key point, and interframe tie point identification errors. These errors tend to compound upstream in the processing workflow during initial image matching and alignment, and may result in downstream derangements in spatial estimation and mesh tessellation.

Visual assessment of the scaled, coregistered endoscopic 3D surface mesh revealed dimensional consistency with rigid landmarks identified on the CT bone model, within expected differences implicit with inclusion of overlying soft tissue and mucosa on the endoscopic model. Despite intentionally inclusive segmentation of $\mathrm{CT}$ image data, margins of the surgical defect were poorly reproduced on the CT bone model, with artifactual uncovering of the internal carotid arteries 

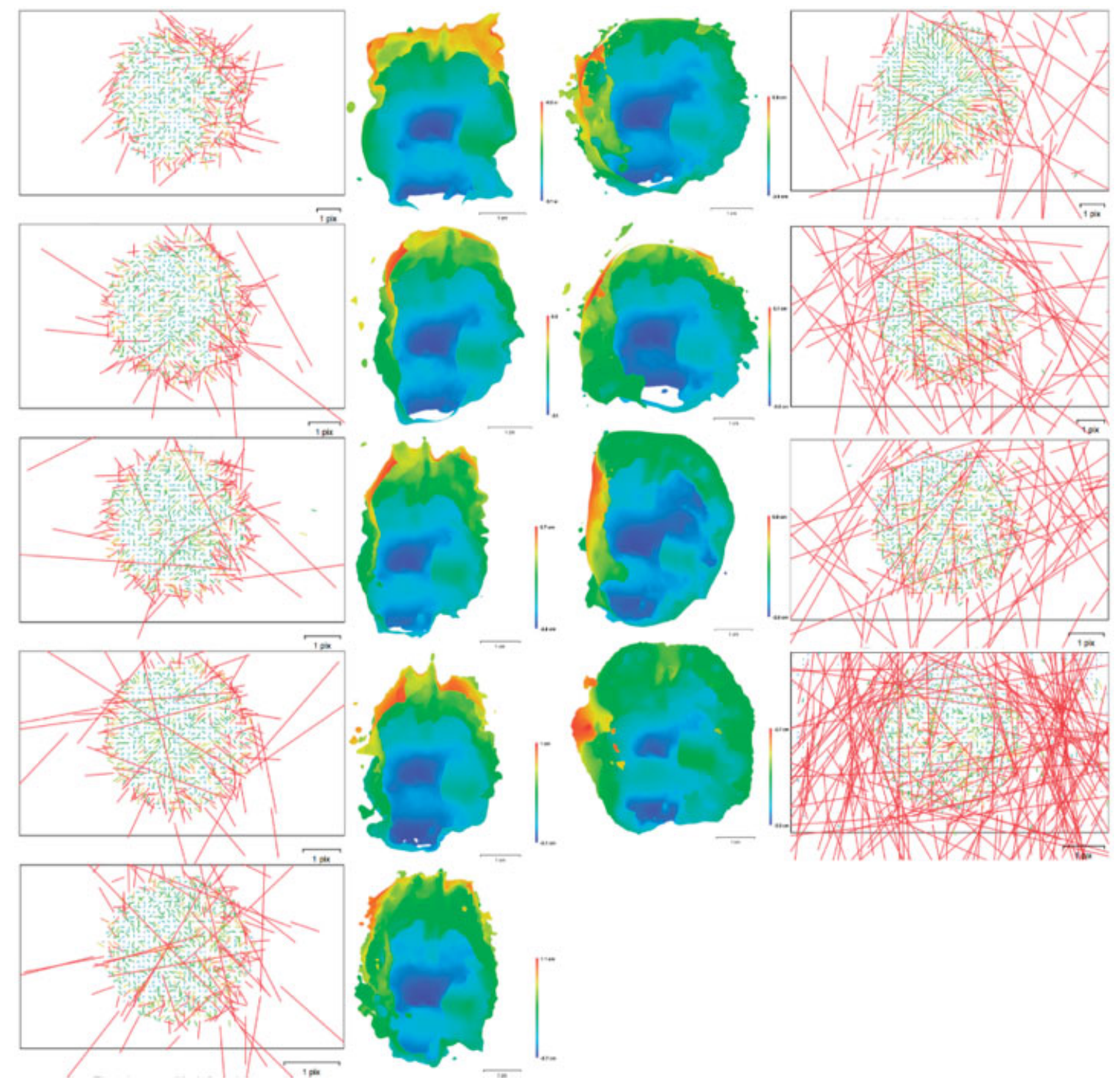

Fig. 4 Average reprojection errors paired with surface depth maps at image batch size reductions of (from top left down): 1:120, 1:60, 1:30, 1:20, 1:10; (from top right down): 1:5, 1:4, 1:3, 1:2. Note that origin points are arbitrarily determined during photogrammetric processing, accounting for slight difference in depth map projection views.

bilaterally appearing contiguous with the defect (-Fig. 3). By contrast, defect margins were preserved on the optical 3D reconstruction, visible as both surface contour and color detail.

\section{Limitations}

This feasibility study is limited primarily by the absence of an anatomic ground truth comparison, planned for inclusion on a subsequent validation study directly examining the adequacy of the resulting gasket-seal using a custom rigid buttress prepared in this manner. As the eventual goal is to assess feasibility of both post-processing and fabrication of a rigid buttress, feasibility of the corresponding fabrication workflow will subsequently be addressed. This entails additional time optimization and biocompatibility considerations specific to fabrication of an implantable gasket-seal buttress which are outside the scope of this postprocessing feasibility study.
Secondary limitations to this study include the absence of repeatability testing for photogrammetric processing which is subject to reprocessing variance related to necessarily arbitrary image and point preselection steps during initial image matching. Additionally, use of a planar calibration object could mask dimensional distortion orthogonal to the surface plane; dimensional fidelity could therefore be more accurately interrogated with a 3D calibration object sufficiently small and sterile that would be introduced into the surgical field. Furthermore, the additional time required to convert video to images, and create a planar buttress model from a mesh model template was not systematically examined, though anecdotally small.

\section{Conclusion}

Operative endoscopic image data acquired during endonasal transsphenoidal resection can be used to directly 3D model visible skull base anatomy using readily available SFM 
photogrammetry software. Endoscopic 3D reconstructions may represent a more reliable depiction of the surgical defect than is available by conventional 3D modeling with CT imaging, owing to preservation of optical color detail with surface contours. The resulting mesh model maintains sufficient dimensional fidelity to facilitate design of a custom gasket-seal buttress model, which can be made available within a narrow operative time window.

\section{References}

1 Garcia-Navarro V, Anand VK, Schwartz TH. Gasket seal closure for extended endonasal endoscopic skull base surgery: efficacy in a large case series. World Neurosurg 2013;80(05):563-568

2 Chambless LB, Mawn LA, Forbes JA, Thompson RC. Porous polyethylene implant reconstruction of the orbit after resection of sphenoorbital meningiomas: a novel technique.J Craniomaxillofac Surg 2012; 40(01):e28-e32

3 Kubota T, Hayashi M, Kabuto M, et al. Reconstruction of the skull base using a silicone plate during transsphenoidal surgery. Surg Neurol 1991;36(05):360-364

4 Sherman JH, Pouratian N, Okonkwo DO, Jane JA Jr, Laws ER. Reconstruction of the sellar dura in transsphenoidal surgery using an expanded polytetrafluoroethylene dural substitute. Surg Neurol 2008;69(01):73-76, discussion 76

5 Kumar A, Maartens NF, Kaye AH. Reconstruction of the sellar floor using Bioglue following transsphenoidal procedures. J Clin Neurosci 2003;10(01):92-95

6 Ridwan-Pramana A, Wolff J, Raziei A, Ashton-James CE, Forouzanfar T. Porous polyethylene implants in facial reconstruction: outcome and complications. J Craniomaxillofac Surg 2015;43 (08):1330-1334

7 Dusick JR, Esposito F, Mattozo CA, Chaloner C, McArthur DL, Kelly DF. Endonasal transsphenoidal surgery: the patient's perspective-survey results from 259 patients. Surg Neurol 2006;65(04):332-341, discussion 341-342

8 Chen HC, Lee ST. Need for intrasellar packing in sellar reconstruction of transsphenoidal surgery: less is more? J Clin Neurosci 2006; 13(04):423-427

9 Seda L, Camara RB, Cukiert A, Burattini JA, Mariani PP. Sellar floor reconstruction after transsphenoidal surgery using fibrin glue without grafting or implants: technical note. Surg Neurol 2006;66 (01):46-49, discussion 49

10 Park J, Guthikonda M. The Medpor sheet as a sellar buttress after endonasal transsphenoidal surgery: technical note. Surg Neurol 2004;61(05):488-492, discussion 493

11 Liebelt BD, Huang M, Baskin DS. Sellar floor reconstruction with the Medpor implant versus autologous bone after transnasal transsphenoidal surgery: outcome in 200 consecutive patients. World Neurosurg 2015;84(02):240-245

12 Shiley SG, Limonadi F, Delashaw JB, et al. Incidence, etiology, and management of cerebrospinal fluid leaks following trans-sphenoidal surgery. Laryngoscope 2003;113(08):1283-1288

13 Berker M, Hazer DB, Yücel T, et al. Complications of endoscopic surgery of the pituitary adenomas: analysis of 570 patients and review of the literature. Pituitary 2012;15(03):288-300

14 Ey-Chmielewska H, Chruściel-Nogalska M, Frączak B. Photogrammetry and its potential application in medical science on the basis of selected literature. Adv Clin Exp Med 2015;24(04):737-741

15 Mendonca DA, Naidoo SD, Skolnick G, Skladman R, Woo AS. Comparative study of cranial anthropometric measurement by traditional calipers to computed tomography and threedimensional photogrammetry. J Craniofac Surg 2013;24(04): $1106-1110$

16 Salazar-Gamarra R, Seelaus R, da Silva JV, da Silva AM, Dib LL, Lauria Dib L. Monoscopic photogrammetry to obtain 3D models by a mobile device: a method for making facial prostheses. J Otolaryngol Head Neck Surg 2016;45(01):33

17 Thormahlen T, Broszio H, Meier PN. Three-dimensional endoscopy. Falk Symp 2002:199-214. doi:2440-43496

18 Leonard S, Reiter A, Sinha A, Ishii M, Taylor RH, Hager GD. Imagebased navigation for functional endoscopic sinus surgery using structure from motion. Proc SPIE Int Soc Opt Eng; 2016

19 Malti A, Bartoli A. Combining conformal deformation and CookTorrance shading for 3-D reconstruction in laparoscopy. IEEE Trans Biomed Eng 2014;61(06):1684-1692

20 Collins T, Bartoli A. 3D reconstruction in laparoscopy with closerange photometric stereo. Med Image Comput Comput Assist Interv 2012;15(Pt 2):634-642

21 Field M, Clarke D, Strup S, Seales WB. Stereo endoscopy as a 3-D measurement tool. Proc 31st Annu Int Conf IEEE Eng Med Biol Soc Eng Futur Biomed EMBC 2009 2009:5748-5751. doi:10.1109/ IEMBS.2009.5332606 